\title{
$K$-analytic versus ccm-analytic sets in nonstandard compact complex manifolds
}

\author{
by \\ Rahim Moosa (Waterloo, ON) and \\ Sergei Starchenko (Notre Dame, IN)
}

\begin{abstract}
It is shown that in an elementary extension of a compact complex manifold $M$, the $K$-analytic sets (where $K$ is the algebraic closure of the underlying real closed field) agree with the ccm-analytic sets if and only if $M$ is essentially saturated. In particular, this is the case for compact Kähler manifolds.
\end{abstract}

1. Introduction and preliminaries. Recall that $\mathbb{R}_{\mathrm{an}}$ is the expansion of the real field by all restricted analytic functions, that is, functions $f$ : $\mathbb{R}^{n} \rightarrow \mathbb{R}(n$ ranging over $\mathbb{N})$ that are identically zero off $[-1,1]^{n}$ and whose restrictions to $[-1,1]^{n}$ are analytic. That $\mathbb{R}_{\text {an }}$ is an o-minimal expansion of the real field follows from Gabrielov's theorem on the complement of a subanalytic set (see [6]).

Let $\mathcal{R}=(\mathbb{R}, \ldots)$ be a fixed o-minimal expansion of $\mathbb{R}_{\text {an }}$ and $M$ a compact complex manifold. We will view the underlying real-analytic manifold of $M$ as living definably in $\mathcal{R}$ and equipped with all the structure induced from $\mathcal{R}$. One way to do so would be to consider $M$ as a definable space in $\mathcal{R}$ in the sense of [7]. However, we proceed more concretely (though less canonically) as follows: the Morrey-Grauert imbedding theorem ([8]) allows us to identify $M$ with a real-analytic submanifold of $\mathbb{R}^{m}$ for some $m \geq 0$. Moreover, by compactness, $M \subseteq \mathbb{R}^{m}$ is globally subanalytic and hence definable in $\mathbb{R}_{\mathrm{an}}$. We therefore can, and do, view $M$ as equipped with the full induced structure from $\mathcal{R}$ : a subset of $M^{n}$ is definable if as a subset of $\mathbb{R}^{m n}$ it is definable in $\mathcal{R}$. For example, every real-analytic subset of $M^{n}$ is definable.

2000 Mathematics Subject Classification: Primary 03C98; Secondary 03C64, 32J27.

Key words and phrases: $K$-analytic set, ccm-analytic set, nonstandard complex manifold.

Rahim Moosa was partially supported by NSERC.

Sergei Starchenko was partially supported by the NSF. 
Now consider the complex manifold structure on $M$. Every complexanalytic subset of $M^{n}$ is real-analytic and hence definable. The complexanalytic structure on $M$ is thus viewed as a reduct of its real-analytic structure. Formally, let us denote by $M_{\mathrm{ccm}}$ this reduct where the underlying set is $M$ and where the language consists of a predicate for each complex-analytic subset of each cartesian power of $M$. A definable subset of $M$ (or its cartesian powers) will be called ccm-definable if it is definable in the reduct $M_{\mathrm{ccm}}$. Recall that $\operatorname{Th}\left(M_{\mathrm{ccm}}\right)$ admits quantifier elimination and is of finite Morley rank ([20]). A more detailed survey of the model theory of $\operatorname{Th}\left(M_{\mathrm{ccm}}\right)$ can be found in [10].

Next, we pass to an elementary extension of the entire situation: let $\widehat{\mathcal{R}}=(R, \ldots)$ be a sufficiently saturated elementary extension of $\mathcal{R}$, and let $\widehat{M} \subseteq R^{m}$ be the interpretation of $M$ in $\widehat{\mathcal{R}}$. We obtain a corresponding elementary extension of $M_{\mathrm{ccm}}$, denoted by $\widehat{M}_{\mathrm{ccm}}$, which is itself a reduct of the induced structure on $\widehat{M}$ from $\widehat{\mathcal{R}}$. So on the "nonstandard" manifold $\widehat{M}$ we have the definable sets, namely those that are definable in $\widehat{\mathcal{R}}$, and the ccm-definable sets, those that are definable in the reduct $\widehat{M}_{\mathrm{ccm}}$.

Notation 1.1. If $\mathcal{N} \preceq \widehat{\mathcal{N}}$ is an elementary extension of first-order structures, and

$$
\mathcal{F}=\left\{F_{a}=\phi(c, a)^{\mathcal{N}}: a \in A=\psi(x)^{\mathcal{N}}\right\}
$$

is a definable family of sets in $\mathcal{N}$, then we let

$$
\widehat{\mathcal{F}}:=\left\{\phi(c, a)^{\widehat{\mathcal{N}}}: a \in \psi(x)^{\widehat{\mathcal{N}}}\right\}
$$

be the corresponding definable family in $\widehat{\mathcal{N}}$. A definable set in $\widehat{\mathcal{N}}$ is then just a member of some $\widehat{\mathcal{F}}$ where $\mathcal{F}$ is a definable family of definable sets in $\mathcal{N}$. The study of definable sets in $\widehat{\mathcal{N}}$ thus amounts to the study of definable families in $\mathcal{N}$.

Among the definable sets in $\widehat{M}$ there are two natural candidates for the "nonstandard complex-analytic" ones. The first of these comes from working entirely in $\operatorname{Th}\left(M_{\mathrm{ccm}}\right)$ :

Definition 1.2. A subset $S \subseteq \widehat{M}^{n}$ is ccm-analytic if there exists a ccm-definable family $\mathcal{F}$ of complex-analytic subsets of $M^{n}$ such that $S \in \widehat{\mathcal{F}}$.

By quantifier elimination for $\operatorname{Th}\left(M_{\mathrm{ccm}}\right)$, every ccm-definable set in $\widehat{M}$ is a finite boolean combination of ccm-analytic sets.

The second notion of "complex-analyticity" in $\widehat{M}$ comes from the work of Y. Peterzil and the second author on complex analysis over algebraically closed fields in o-minimal structures. Let $R$ be a real closed field and $K$ its algebraic closure. In [14-16] Y. Peterzil and the second author investigate differentiability with respect to the field $K$ for functions of several variables 
definable in o-minimal expansions of $R$. Their analysis leads to a notion of $K$-analytic set. We will not give their definition here as we will be concerned with only a special case. Suffice it to say that $K$-analyticity is definable in parameters (see Corollary 4.13 of [14]), and that if $K=\mathbb{C}$ and one is working in an o-minimal expansion of $\mathbb{R}_{\mathrm{an}}$ then $K$-analytic is just complex-analytic. It follows that if $R$ is the underlying real closed field of an elementary extension of an o-minimal expansion of $\mathbb{R}_{\mathrm{an}}$, then the notion of $K$-analytic subset of $\widehat{M}$ introduced in [16] has the following more direct characterisation-which will serve as a definition for us.

Definition 1.3. Let $R$ be the underlying real closed field of $\widehat{\mathcal{R}}$ and $K$ its algebraic closure. A subset $S \subseteq \widehat{M}^{n}$ is $K$-analytic if there exists a definable family $\mathcal{F}$ of complex-analytic subsets of $M^{n}$ such that $S \in \widehat{\mathcal{F}}$.

In particular, every ccm-analytic set is $K$-analytic. Our purpose in this note is to characterise the class of compact complex manifolds $M$ for which the converse holds.

EXAMPle 1.4. Consider the case when $M$ is a projective algebraic variety $V$ (or rather the set of $\mathbb{C}$-rational points of $V$ ). Then $\widehat{M}$ can be identified with the $K$-rational points of $V$. Moreover, by quantifier elimination and Chow's theorem, every ccm-definable family of complex-analytic subsets of $M^{n}$ is a Zariski-constructible family of algebraic subsets of $M^{n}$. It follows that the ccm-analytic subsets of $\widehat{M}^{n}$ in this case are exactly the algebraic subsets over $K$. Now, in [14] Y. Peterzil and the second author prove a nonstandard version of Chow's theorem so that the $K$-analytic subsets of $\widehat{M}^{n}$ are also just the algebraic subsets over $K$. Hence, for $M$ a projective variety, $K$-analyticity and ccm-analyticity coincide in $\widehat{M}$.

EXAmple 1.5 (see Section 3.3 of [16]). Fix real numbers $a$ and $b$ lying strictly between 0 and 1 , and consider the Hopf surface $M$ obtained as the quotient of $\mathbb{C}^{2} \backslash\{(0,0)\}$ by the action of the group of automorphisms generated by $(z, w) \mapsto(a z, b w)$. It is explained in [18] that there are no infinite ccm-definable families of automorphisms of $M$. Hence $\widehat{M}$ has no ccm-analytic automorphisms (i.e., no automorphims whose graph is a ccmanalytic subset of $\widehat{M}^{2}$ ) other than the interpretations of the complex-analytic automorphisms of $M$ itself. On the other hand, as pointed out in [16], it is not hard to construct infinite definable (in $\mathcal{R}$ ) families of complex-analytic automorphisms of $M$. This gives rise to $K$-analytic subsets of $\widehat{M}^{2}$ that are not ccm-analytic.

The characterisation we obtain will be in terms of the following notion introduced by the first author in [11]: a compact complex manifold $M$ is essentially saturated if there exists a countable collection of complex-analytic subsets of $M$ and its cartesian powers, $\mathcal{L}_{0}$, such that every ccm-definable 
set is definable (with parameters) in the language where there is a predicate for each complex-analytic set in $\mathcal{L}_{0}$. The terminology comes from the fact (Proposition 2.3 of [11]) that if $M$ is essentially saturated and $\mathcal{L}_{0}$ is as above, then the structure $\left(M, \mathcal{L}_{0}\right)$ is saturated, and definabilities in $\left(M, \mathcal{L}_{0}\right)$ and $M_{\mathrm{ccm}}$ agree.

Our main observation is the following result which we prove in Section 3.

THEOREM 1.6. The following are equivalent:

(a) $M$ is essentially saturated.

(b) The $K$-analytic and ccm-analytic subsets of $\widehat{M}^{n}$, for all $n>0$, coincide.

Since compact Kähler manifolds are essentially saturated (see Section 4 of [11]), we obtain:

COROLlary 1.7. If $M$ is a compact Kähler manifold then every $K$ analytic subset of $\widehat{M}^{n}$ is ccm-analytic for all $n>0$.

We would like to thank Kobi Peterzil and Patrick Speissegger with whom we had several discussions that led to the observations contained in this paper.

2. The Barlet space, essential saturation, and volume. In [11] essential saturation is given a geometric characterisation in terms of certain universal families of analytic subspaces. In this section we will recall Barlet's space of compact cycles of a complex manifold, recast essential saturation as a condition on the Barlet spaces, and discuss a theorem of Lieberman's which will be used in the proof of Theorem 1.6. A more detailed exposition of this material can be found in Section 3 of [12].

For $M$ any complex manifold, a $k$-cycle of $M$ is a finite linear combination $Z=\sum_{i} n_{i} Z_{i}$ where the $Z_{i}$ 's are distinct $k$-dimensional irreducible compact complex-analytic subsets of $M$, and each $n_{i}$ is a positive integer called the multiplicity of $Z_{i}$ in $Z$. By $|Z|$ we mean the underlying set or support of $Z$, namely $\bigcup_{i} Z_{i}$. We denote the set of all $k$-cycles of $M$ by $\mathcal{B}_{k}(M)$, and the set of all cycles of $M$ by $\mathcal{B}(M):=\bigcup_{k} \mathcal{B}_{k}(M)$. In [1] Barlet endowed $\mathcal{B}_{k}(M)$ with a natural structure of a complex-analytic space whereby if for $s \in \mathcal{B}_{k}(M)$ we let $Z_{s}$ denote the cycle respresented by $s$, then the set $\{(s, x)$ : $\left.s \in \mathcal{B}_{k}(M), x \in\left|Z_{s}\right|\right\}$ is a complex-analytic subset of $\mathcal{B}_{k}(M) \times M$. Equipped with this complex structure, $\mathcal{B}(M)$ is called the Barlet space of $M$. When $M$ is a projective variety the Barlet space coincides with the Chow scheme. In [3] it is shown that

$$
\mathcal{B}^{*}(M):=\left\{s \in \mathcal{B}(M): Z_{s} \text { is irreducible with multiplicity } 1\right\}
$$

is a Zariski open subset of $\mathcal{B}(M)$ : its complement in $\mathcal{B}(M)$ is a proper 
complex-analytic subset. An irreducible component of $\mathcal{B}(M)$ is prime if it has nonempty intersection with $\mathcal{B}^{*}(M)$.

The characterisation of essential saturation referred to above can be expressed in terms of the Barlet space as follows.

FACT 2.1 (Moosa [11]). Suppose $M$ is a compact complex manifold. Then $M$ is essentially saturated if and only if every prime component of $\mathcal{B}\left(M^{n}\right)$ is compact, for all $n>0$.

Actually, in Theorem 3.3 of [11] this is done with the universal family of analytic subspaces - the Douady space - rather than cycles. However, the same arguments work for Barlet spaces as well $\left(^{1}\right)$. Or alternatively, since there is a holomorphic map from the Douady space to the Barlet space whose image contains $\mathcal{B}^{*}(M)$ (see Chapitre $\mathrm{V}$ of [1]), compactness of the components of the Douady space implies compactness of the prime components of the Barlet space.

The main technique for determining whether a prime component of the Barlet space is compact comes from a theorem of Lieberman's which involves some hermitian geometry. Recall that if $M$ is a complex manifold then there is a one-to-one correspondence between hermitian metrics and positive real $(1,1)$-forms on $M$, given by $h \mapsto \omega:=-\operatorname{Im}(h)$. Moreover, $\operatorname{Re}(h)$ is a riemannian metric on $M$. Wirtinger's theorem allows us to compute the volume of a compact complex submanifold of $M$ with respect to this riemannian metric (denoted by $\mathrm{vol}_{h}$ ) by integrating the appropriate exterior power of the associated (1,1)-form over the submanifold: if $Z \subseteq M$ is a compact complex submanifold of complex dimension $k$, then the volume of $Z$ is given by

$$
\operatorname{vol}_{h}(Z)=\frac{1}{k !} \int_{Z} \omega^{k}
$$

where $\omega^{k}$ is the $k$ th exterior power of $\omega$ (see Section 3.1 of [19]).

If $Z$ is a possibly singular complex-analytic subset (irreducible, compact, of dimension $k$ ), then equation (2.1) can serve as the definition of volume; it agrees with the volume of the regular locus of $Z$. More generally, if $Z=\sum_{i} n_{i} Z_{i}$ is a $k$-cycle of $M$, then the volume of $Z$ with respect to $h$ is $\operatorname{vol}_{h}(Z):=\sum_{i} n_{i} \operatorname{vol}_{h}\left(Z_{i}\right)$.

Note that taking volumes of cycles induces a function $\operatorname{vol}_{h}: \mathcal{B}(M) \rightarrow \mathbb{R}$ given by $\operatorname{vol}_{h}(s):=\operatorname{vol}_{h}\left(Z_{s}\right)$.

FACT 2.2 (Lieberman [9]). Suppose $M$ is a compact complex manifold equipped with a hermitian metric $h$, and $X$ is any subset of $\mathcal{B}(M)$. Then $X$ is relatively compact in $\mathcal{B}(M)$ if and only if $\mathrm{vol}_{h}$ is bounded on $X$.

$\left({ }^{1}\right)$ Indeed it is easier as one can replace the use of Hironaka's flattening theorem by the much simpler geometric flattening theorem of [2]. 
One application of this fact is to show that compact Kähler manifolds are essentially saturated (see Section 3 of [12]). We will use Lieberman's theorem together with the following consequence of a result of van den Dries' to prove Theorem 1.6.

Proposition 2.3. Suppose $M$ is a compact complex manifold (viewed as a definable space in an o-minimal expansion $\mathcal{R}$ of $\mathbb{R}_{\mathrm{an}}$ ) and $h$ is a hermitian metric on $M$. If $\mathcal{F}$ is a definable family of complex-analytic subsets of $M$ then $\left\{\operatorname{vol}_{h}(F): F \in \mathcal{F}\right\}$ is bounded.

Proof. In the e-print [13] there is an argument for how this follows from the fact (Proposition 4.1 of [5]) that Hausdorff measure in definable families is bounded. For the sake of completeness we include it here.

Let $\mathcal{F}=\left\{F_{a}: a \in A\right\}$. Since the complex dimension is twice the real dimension and the latter is definable in parameters by o-minimality, we may assume that there is a $k \geq 0$ such that $\operatorname{dim}_{\mathbb{C}}\left(F_{a}\right)=k$ for all $a \in A$. By compactness of $M$ it suffices to show that for each $p \in M$ there is an open neighbourhood $V$ of $p$ such that $\operatorname{vol}_{h}\left(F_{a} \cap V\right)$ is bounded as $a$ varies in $A$.

Consider a chart $\alpha: V \rightarrow D$ at $p$ such that $D$ is a definable bounded open ball in $\mathbb{C}^{m}$ (where $\left.m=\operatorname{dim}_{\mathbb{C}}(M)\right)$ and $\alpha(p)=0$. Then $\left.\operatorname{Re}(h)\right|_{V}$ induces via $\alpha$ a riemannian metric $g$ on $D$. For each $d \in D$, let $r_{d}$ be the maximum of $g_{d}(v, v)$ for all $v$ in the closed unit sphere in the tangent space $T_{d} D$ of $D$ at $d$. By the continuity of the map $d \mapsto r_{d}$, for a smaller open ball $D^{\prime}$ compactly contained in $D$, there is an $r>0$ such that $r_{d} \leq r$ for all $d \in D^{\prime}$. Shrinking the chart if necessary we may assume $D^{\prime}=D$. Replacing $\alpha$ with $\alpha / \sqrt{r}$, we can now assume that for all $d \in D$ and all $v \in T_{d} D, g_{d}(v, v) \leq\|v\|_{d}^{2}$ where || $\|$ denotes the standard norm. In other words, the length of tangent vectors with respect to the riemannian metric induced on $D$ from $\left.h\right|_{V}$ is not greater than the length with respect to the standard metric. It follows that the volume of $\alpha\left(F_{a} \cap V\right)$ with respect to the standard riemannian metric on $D$ is not less than $\operatorname{vol}_{h}\left(F_{a} \cap V\right)$, for any $a \in A$. But the volume of $\alpha\left(F_{a} \cap V\right)$ with respect to the standard riemannian metric is equal to the $2 k$-dimensional Hausdorff measure of $\alpha\left(F_{a} \cap V\right)$. Since $\left\{F_{a} \cap V: a \in A\right\}$ is a definable family, the $2 k$-dimensional Hausdorff measure of $\alpha\left(F_{a} \cap V\right)$ is bounded as $a$ varies in $A$ by Proposition 4.1 of [5]. Hence, $\operatorname{vol}_{h}\left(F_{a} \cap V\right)$ is bounded as $a$ varies in $A$, as desired.

3. Proof of Theorem 1.6. We begin with a characterisation of ccmanalytic subsets that is more along the lines of $[17,10]$. Let $\mathcal{A}$ be the manysorted structure where there is a sort for each compact complex-analytic space (not necessarily smooth) and the language consists of a predicate for each complex-analytic subset of each cartesian product of sorts. Then $\operatorname{Th}(\mathcal{A})$ admits quantifier elimination and is of finite Morley rank (sort by sort). If 
$M$ is a compact complex manifold then $M$ is a sort of $\mathcal{A}$ and $M_{\mathrm{ccm}}$ is just the structure induced on $M$ by $\mathcal{A}$. The whole many-sorted structure $\mathcal{A}$ is interpretable in $\mathcal{R}$. Hence there is a corresponding elementary extension $\widehat{\mathcal{A}}$ of $\mathcal{A}$ interpretable in $\widehat{\mathcal{R}}$.

Lemma 3.1. A subset $S \subseteq \widehat{M}^{n}$ is ccm-analytic if and only if there exist:

- an irreducible compact complex-analytic space $B$,

- a complex-analytic subset $Z \subseteq B \times M^{n}$,

- a generic point e of $B$ in $\widehat{\mathcal{A}}$,

such that $S$ is the fibre of $Z$ above e in $\widehat{\mathcal{A}}$.

Proof. This is discussed in detail in Section 6 of [10], but we give some explanations here. First of all, suppose $S$ is the generic fibre of $Z \rightarrow B$ where $B$ and $Z \subseteq B \times M^{n}$ are as in the lemma. Note that $\mathcal{F}:=\left\{Z_{b}: b \in B\right\}$ is a family of complex-analytic subsets of $M^{n}$ definable in $\mathcal{A}$. By definability of types, $\mathcal{F}$ is actually definable with parameters from $M$ itself. Hence $\mathcal{F}$ is a ccm-definable family of complex-analytic subsets of $M^{n}$. Since $S \in \widehat{\mathcal{F}}, S$ is ccm-analytic.

For the converse, suppose $S \in \widehat{\mathcal{F}}$ for some ccm-definable family of complex-analyitc subsets of $M^{n}$. Then there are ccm-definable sets $E \subseteq M^{l}$ and $F \subseteq E \times M^{n}$ whose fibres over $E$ are complex-analytic, and such that $S=F_{e}$ for some $e \in E\left(\widehat{M}_{\mathrm{ccm}}\right)$. Let $a \in S$ be a generic point of $S$ over $e$ (so $a$ is not contained in any proper $e$-definable ccm-analytic subset of $S)$. Let $B:=\operatorname{locus}(e)$ be the smallest complex-analytic subset of $M^{l}$ with $e \in B(\widehat{M})$. Let $Z:=\operatorname{locus}(e, a) \subseteq B \times M^{n}$. Then it is not hard to see that $S=Z_{e}$

Proof of Theorem 1.6. Let us recall the set-up of the theorem:

- $M$ is a compact complex manifold viewed as a definable set in a fixed o-minimal expansion $\mathcal{R}$ of $\mathbb{R}_{\text {an }}$.

- $\widehat{\mathcal{R}}$ is a sufficiently saturated elementary extension of $\mathcal{R}$ with underlying real closed field $R$ whose algebraic closure is $K$.

- $\widehat{M}$ is the corresponding elementary extension of $M$ viewed as a definable set in $\widehat{\mathcal{R}}$.

We first show (a) implies (b): if $M$ is essentially saturated then every $K$-analytic subset of $\widehat{M}^{n}$ is ccm-analytic, for all $n>0$. So assume that $M$ is essentially saturated and let $S \subseteq \widehat{M}^{n}$ be $K$-analytic. Let $\mathcal{F}=\left\{F_{a}: a \in A\right\}$ be a definable family of complex-analytic subsets of $M^{n}$ such that $S \in \widehat{\mathcal{F}}$.

First of all, by the definable trivialisation theorem in o-minimal structures (see Chapter 9 of [7]) the connected components of $\operatorname{reg}\left(F_{a}\right)$, and hence the irreducible components of $F_{a}$, are uniformly definable. By taking unions it therefore suffices to consider the case when each $F_{a}$ is irreducible. 
Hence, for each $a \in A$ there is a unique $b \in \mathcal{B}^{*}\left(M^{n}\right)$ such that $F_{a}=Z_{b}$. Let $X \subseteq \mathcal{B}^{*}\left(M^{n}\right)$ be the set of such $b$ 's. Now fix a hermitian metric $h$ on $M^{n}$. By Proposition 2.3, $\left\{\operatorname{vol}_{h}\left(F_{a}\right): a \in A\right\}$ is bounded, and hence $\operatorname{vol}_{h}$ is bounded on $X$. It follows that $X$ is relatively compact in $\mathcal{B}\left(M^{n}\right)$ (this is Fact 2.2). So $X$ is contained in the union of finitely many prime components of $\mathcal{B}\left(M^{n}\right)$, say $B:=\bigcup_{i=1}^{l} B_{i}$. By essential saturation, each $B_{i}$ is compact (this is Fact 2.1). Hence $\mathcal{G}:=\left\{Z_{b}: b \in B \cap \mathcal{B}^{*}\left(M^{n}\right)\right\}$ is a ccm-definable family of complex-analytic subsets of $M^{n}$ (see Lemma 3.1). Since $X \subseteq B \cap \mathcal{B}^{*}\left(M^{n}\right)$, every member of $\mathcal{F}$ is in $\mathcal{G}$. It follows that every member of $\widehat{\mathcal{F}}$ is in $\widehat{\mathcal{G}}$. In particular $S \in \widehat{\mathcal{G}}$, and so $S$ is ccm-analytic as desired.

For the converse assume that $M$ is not essentially saturated. By Fact 2.1, for some $n>0$, there exists a noncompact prime component $B_{0}$ of $\mathcal{B}\left(M^{n}\right)$. Let $B_{1}:=\operatorname{reg}\left(B_{0}\right) \cap \mathcal{B}^{*}\left(M^{n}\right)$. Then $B_{1}$ is a nonempty Zariski open subset of $B_{0}$ and is a complex-analytic manifold. Using the Morrey-Grauert imbedding theorem we may assume that $B_{1} \subseteq \mathbb{R}^{l}$ for some $l \geq 0$, and that as a real-analytic manifold it is a submanifold of $\mathbb{R}^{l}$. Since $G_{0}:=\left\{(s, x): s \in B_{0}\right.$, $\left.x \in\left|Z_{s}\right|\right\}$ is a complex-analytic subset of $B_{0} \times M^{n}, G_{1}:=\left\{(s, x): s \in B_{1}\right.$, $\left.x \in\left|Z_{s}\right|\right\}$ is real-analytic as a subset of $\mathbb{R}^{l} \times \mathbb{R}^{m n}$. Now fix an arbitrary $a \in B_{1}$, choose an open ball $V \subseteq \mathbb{R}^{l}$ of radius one centred at $a$, and set $D:=V \cap B_{1}$. Then $G_{1} \cap\left(D \times \mathbb{R}^{m n}\right)$ is a real-analytic subset of $\mathbb{R}^{l} \times \mathbb{R}^{m n}$ which is relatively compact (being contained in $\left.D \times M^{n}\right)$. Hence $G_{1} \cap\left(D \times \mathbb{R}^{m n}\right)$ is definable in $\mathbb{R}_{\text {an }}$. It follows that $\mathcal{F}:=\left\{Z_{d}: d \in D\right\}$ is definable in $\mathcal{R}$. The point here is that this definable family of complex-analytic subsets of $M^{n}$ has as its parameter space a nonempty open subset of the noncompact prime component $B_{0}$. We will show that this forces some member of $\widehat{\mathcal{F}}$ to be non-ccm-analytic. (Note that $\mathcal{F}$ itself fails to be ccm-definable because $D$ is not ccm-definable.)

ClAim 3.2. If $S \in \widehat{\mathcal{F}}$ is ccm-analytic then there exists a compact complexanalytic subset $C \subseteq \mathcal{B}\left(M^{n}\right)$ such that $S \in \widehat{\mathcal{G}}$ where $\mathcal{G}:=\left\{Z_{c}: c \in C \cap\right.$ $\left.\mathcal{B}^{*}\left(M^{n}\right)\right\}$.

Proof of Claim. By Lemma 3.1, there exists an irreducible compact complex-analytic set $A$ and a complex-analytic subset $F \subseteq A \times M^{n}$ such that $S$ is a generic fibre of $F$ over $A$ in $\widehat{\mathcal{A}}$. Note that since $S \in \overline{\widehat{\mathcal{F}}}$, every nonempty Zariski open subset of $A$ contains an element $a$ such that $F_{a} \in \mathcal{F}$. As the members of $\mathcal{F}$ are irreducible, it follows that over a nonempty Zariski open subset of $A$ the fibres of $F$ are irreducible. In particular, there is a unique irrreducible component of $F$ that projects onto $A$ and which contains the general fibres of $F$, and hence we may replace $F$ by this irreducible component. Note also that after possibly shrinking the Zariski open set, these general fibres are of constant dimension. It follows by the universal property of the Barlet space (together with the geometric flattening theorem of [2]) 
that there exists a meromorphic map $g: A \rightarrow \mathcal{B}\left(M^{n}\right)$ and a nonempty Zariski open subset $U \subseteq A$ such that for all $a \in U, g(a) \in \mathcal{B}^{*}\left(M^{n}\right)$ and $F_{a}=Z_{g(a)}$ (see for example Proposition 2.20 of [4]). Let $C$ be the closure of the image of $U$ under $g$ - which will be an irreducible compact complexanalytic subset of $\mathcal{B}\left(M^{n}\right)$ since $A$ is compact and $g$ is meromorphic - and let $\mathcal{G}:=\left\{Z_{c}: c \in C \cap \mathcal{B}^{*}\left(M^{n}\right)\right\}$. Since $S$ is a generic fibre of $F$ over $A, S \in \widehat{\mathcal{G}}$.

Suppose for a contradiction that every member of $\widehat{\mathcal{F}}$ is ccm-analytic. By saturation and the claim, $\mathcal{F}$ must be covered by finitely many families of the form given by the claim. Taking unions this means that there exists a compact complex-analytic subset $C \subseteq \mathcal{B}\left(M^{n}\right)$ such that every member of $\mathcal{F}$ is contained in $\mathcal{G}:=\left\{Z_{c}: c \in C \cap \mathcal{B}^{*}\left(M^{n}\right)\right\}$. But this means that $D \subseteq C \cap B_{0}$. By construction, $D$ is a nonempty open subset of $B_{0}$ while $C \cap B_{0}$ is a complex-analytic subset of the irreducible complex-analytic space $B_{0}$. Hence $B_{0}=C \cap B_{0}$, and so $B_{0}$ is an irreducible component of $C$. But this contradicts the fact that $C$ is compact while $B_{0}$ is not. There must therefore exist some member of $\widehat{\mathcal{F}}$ that is not ccm-analytic. We have shown that not every $K$-analytic set is ccm-analytic, as desired.

This completes the proof of Theorem 1.6.

\section{References}

[1] D. Barlet, Espace analytique réduit des cycles analytiques complexes compacts d'un espace anlytique complexe de dimension finie, in: Fonctions de plusieurs variables complexes, II (Sém. François Norguet, 1974-1975), Lecture Notes in Math. 482, Springer, 1975, 1-158.

[2] - Majoration du volume des fibres génériques et forme géométrique du théorème d'aplatissement, C. R. Acad. Sci. Paris. Sér. A-B 288 (1979), A29-A31.

[3] F. Campana, Application de l'espace des cycles à la classification biméromorphe des espaces analytiques Kählériens compacts, $\mathrm{PhD}$ thesis, Univ. Nancy 1, Prépublication de l'Univ. Nancy 1, no. 2, 1980, 163 pp.

[4] F. Campana and T. Peternell, Cycle spaces, in: H. Grauert, T. Peternell, and R. Remmert (eds.), Several Complex Variables VII, Encyclopedia Math. Sci. 74, Springer, Berlin, 1994, 319-349.

[5] L. van den Dries, Limit sets in o-minimal structures, preprint.

[6] - , A generalization of the Tarski-Seidenberg theorem, and some nondefinability results, Bull. Amer. Math. Soc. (N.S.) 15 (1986), 189-193.

[7] -, Tame Topology and o-Minimal Structures, Cambridge Univ. Press, 1998.

[8] H. Grauert, On Levi's problem and the imbedding of real-analytic manifolds, Ann. of Math. (2) 68 (1958), 460-472.

[9] D. Lieberman, Compactness of the Chow scheme: applications to automorphisms and deformations of Kähler manifolds, in: Fonctions de plusieurs variables complexes, III (Sém. François Norguet, 1975-1977), Lecture Notes in Math. 670, Springer, 1978, 140-186.

[10] R. Moosa, The model theory of compact complex spaces, in: Logic Colloquium '01, Lecture Notes in Logic 20, Assoc. Symbol. Logic, 2005, 317-349. 
[11] R. Moosa, On saturation and the model theory of compact Kähler manifolds, J. Reine Angew. Math. 586 (2005), 1-20.

[12] R. Moosa and A. Pillay, Model theory and Kähler geometry, in: Model Theory with Applications to Algebra and Analysis, Z. Chatzidakis et al. (eds.), Cambridge Univ. Press, to appear.

[13] R. Moosa, P. Speissegger, and S. Starchenko, Compactness of cycle spaces and definabiltiy in o-minimal expansions of $\mathbb{R}_{\mathrm{an}}$, http://www.newton.cam.ac.uk/preprints/ NI05040.pdf, 2005.

[14] Y. Peterzil and S. Starchenko, Complex analytic geometry in a nonstandard setting, in: Model Theory with Applications to Algebra and Analysis, Z. Chatzidakis et al. (eds.), Cambridge Univ. Press, to appear.

[15] -, -, Expansions of algebraically closed fields in o-minimal structures, Selecta Math. 7 (2001), 409-445.

[16] - - - Expansions of algebraically closed fields II: functions of several variables, J. Math. Logic 3 (2003), 1-35.

[17] A. Pillay, Some model theory of compact complex spaces, in: Hilbert's Tenth Problem: Relations with Arithmetic and Algebraic Geometry (Ghent, 1999), Contemp. Math. 270, Amer. Math. Soc., 2000, 323-338.

[18] A. Pillay and T. Scanlon, Meromorphic groups, Trans. Amer. Math. Soc. 355 (2003), 3843-3859.

[19] C. Voisin, Hodge Theory and Complex Algebraic Geometry, I, Cambridge Stud. Adv. Math. 76, Cambridge Univ. Press, 2002.

[20] B. Zilber, Model theory and algebraic geometry, in: Proc. 10th Easter Conference on Model Theory, Humboldt Univ., Berlin, 1993, 202-222.

Department of Pure Mathematics

University of Waterloo

200 University Avenue West

Waterloo, Ontario, Canada N2L 3G1

E-mail: rmoosa@math.uwaterloo.ca
Department of Mathematics University of Notre Dame 255 Hurley Hall Notre Dame, IN 46556-4618, U.S.A. E-mail: starchenko.1@nd.edu 